\title{
Supplement
}

Table S1: Uncertainty classes as defined for the routine daily analysis at ARSO and corresponding numbers of routinely picked first arrival times. All arrival times were picked by hand and uncertainty intervals subjectively determined by the analysts.

\begin{tabular}{|l|l|l|}
\hline Uncertainty class & Uncertainty time interval $[\mathrm{s}]$ & Number of first P, S arrival times \\
\hline 0 & $<0.1$ & $70,059(\mathrm{P}), 37,187(\mathrm{~S})$ \\
\hline 1 & $0.1-0.2$ & $21,849(\mathrm{P}), 23,914(\mathrm{~S})$ \\
\hline 2 & $0.2-0.5$ & $33,496(\mathrm{P}), 38,814(\mathrm{~S})$ \\
\hline 3 & $0.5-1.0$ & $2,504(\mathrm{P}), 3,157(\mathrm{~S})$ \\
\hline 4 & $>1.0$ & $1,565(\mathrm{P}), 1,944(\mathrm{~S})$ \\
\hline 9 & clock error & $777(\mathrm{P}$ and $\mathrm{S})$ \\
\hline Total & & $130,247(\mathrm{P}), 105,019(\mathrm{~S})$ \\
\hline
\end{tabular}


Initial 1-D models with final velocity models

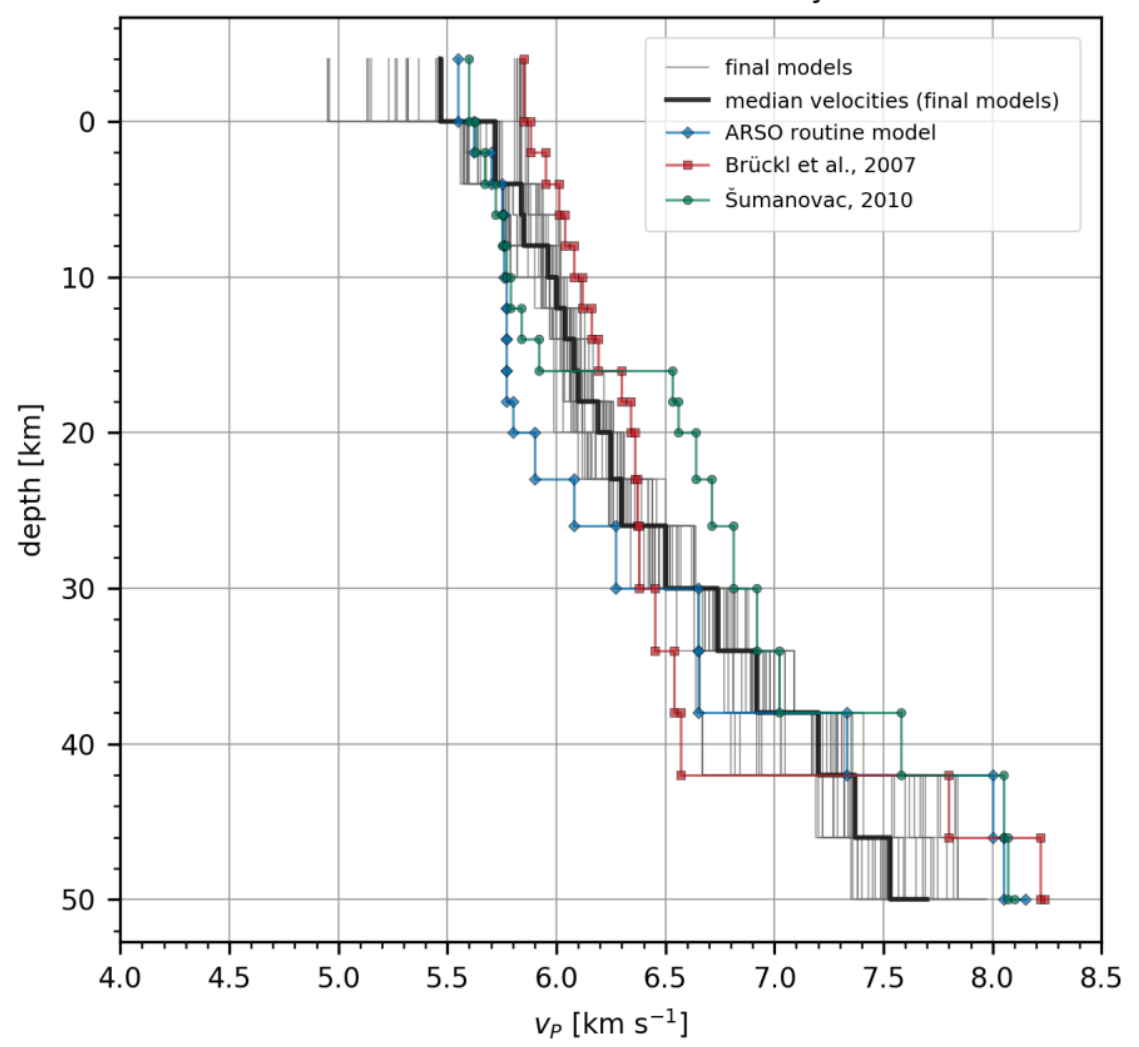

Figure S2: Final regional velocity models obtained for the final layering of a priori initial models, final set of inversion parameters, and variable total number of iterations. Also shown are the a priori initial models and the median velocities. 
Table S3: Velocities and statistics obtained for the regional inversion. Models used to calculate the statistical parameters are shown in Fig. S2.

\begin{tabular}{|c|c|c|c|c|c|c|}
\hline Depth [km] & $\mathrm{M} 1\left[\mathrm{~km} \mathrm{~s}^{-1}\right]$ & $\mathrm{M} 2\left[\mathrm{~km} \mathrm{~s}^{-1}\right]$ & Median $\left[\mathrm{km} \mathrm{s}^{-1}\right]$ & 15 th perc. $\left[\mathrm{km} \mathrm{s}^{-1}\right]$ & 85th perc. $\left[\mathrm{km} \mathrm{s}^{-1}\right]$ & Delta perc. $\left[\mathrm{km} \mathrm{s}^{-1}\right]$ \\
\hline-4 & 5.23 & 5.32 & 5.47 & 5.17 & 5.84 & 0.67 \\
\hline 0 & 5.58 & 5.57 & 5.72 & 5.58 & 5.84 & 0.26 \\
\hline 2 & 5.59 & 5.57 & 5.72 & 5.59 & 5.84 & 0.25 \\
\hline 4 & 5.78 & 5.76 & 5.84 & 5.76 & 5.92 & 0.16 \\
\hline 6 & 5.78 & 5.77 & 5.85 & 5.77 & 5.94 & 0.17 \\
\hline 8 & 5.93 & 5.93 & 5.96 & 5.88 & 6 & 0.12 \\
\hline 10 & 5.93 & 5.95 & 6 & 5.95 & 6.02 & 0.07 \\
\hline 12 & 6.02 & 6.03 & 6.04 & 5.98 & 6.08 & 0.1 \\
\hline 14 & 6.03 & 6.06 & 6.08 & 6.03 & 6.11 & 0.08 \\
\hline 16 & 6.04 & 6.08 & 6.1 & 6.06 & 6.15 & 0.09 \\
\hline 18 & 6.13 & 6.08 & 6.19 & 6.08 & 6.25 & 0.17 \\
\hline 20 & 6.14 & 6.14 & 6.25 & 6.15 & 6.3 & 0.15 \\
\hline 23 & 6.3 & 6.32 & 6.3 & 6.26 & 6.42 & 0.16 \\
\hline 26 & 6.49 & 6.38 & 6.5 & 6.43 & 6.61 & 0.18 \\
\hline 30 & 6.63 & 6.72 & 6.74 & 6.68 & 6.82 & 0.14 \\
\hline 34 & 6.92 & 6.91 & 6.92 & 6.81 & 7.02 & 0.21 \\
\hline 38 & 7.17 & 7.18 & 7.2 & 6.86 & 7.3 & 0.44 \\
\hline 42 & 7.2 & 7.22 & 7.37 & 7.27 & 7.69 & 0.42 \\
\hline 46 & 7.36 & 7.38 & 7.53 & 7.44 & 7.74 & 0.3 \\
\hline 50 & 7.5 & 7.52 & 7.7 & 7.59 & 7.89 & 0.3 \\
\hline
\end{tabular}




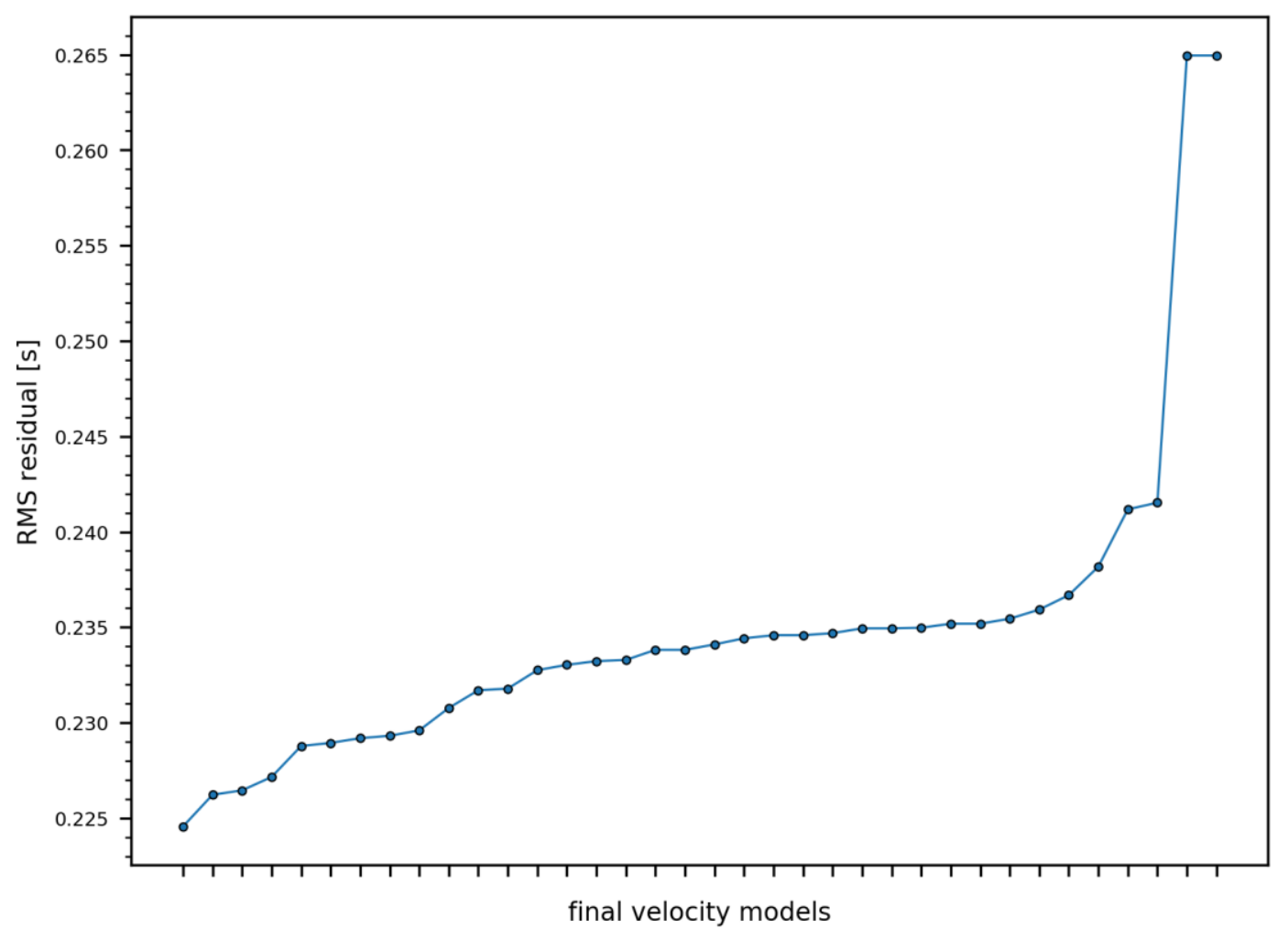

Figure S4: Root mean square residuals obtained after inversions for the final regional models (Fig. S2) in increasing order. 


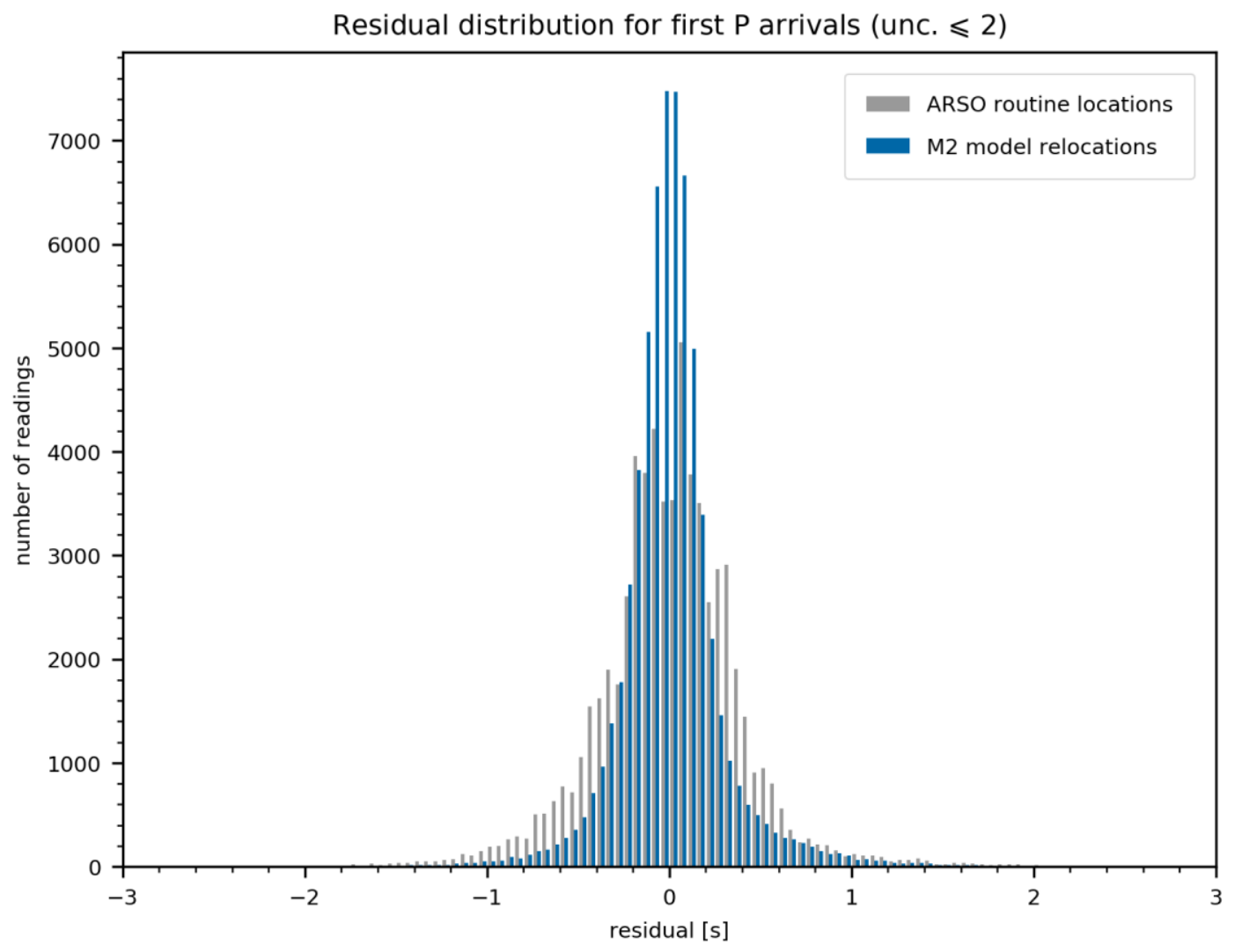

20 Figure S5: Comparison of the residual distributions before and after the relocation of the earthquakes shown in Fig. 11. 


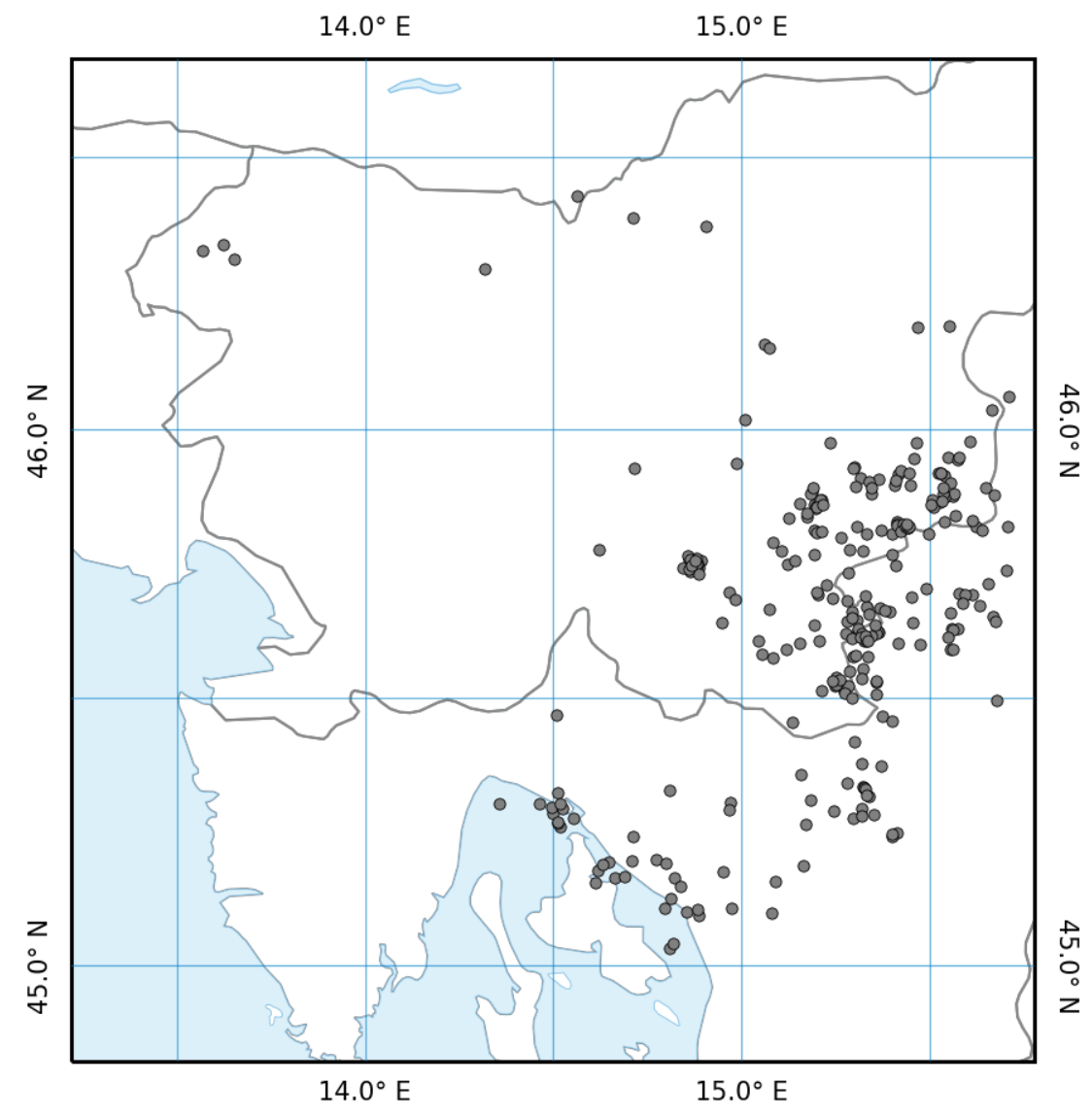

Figure S6: Map of 280 events relocated to depths above $1.0 \mathrm{~km}$ with the M2 velocity model. 


\section{DEPTH $[\mathrm{km}]$}
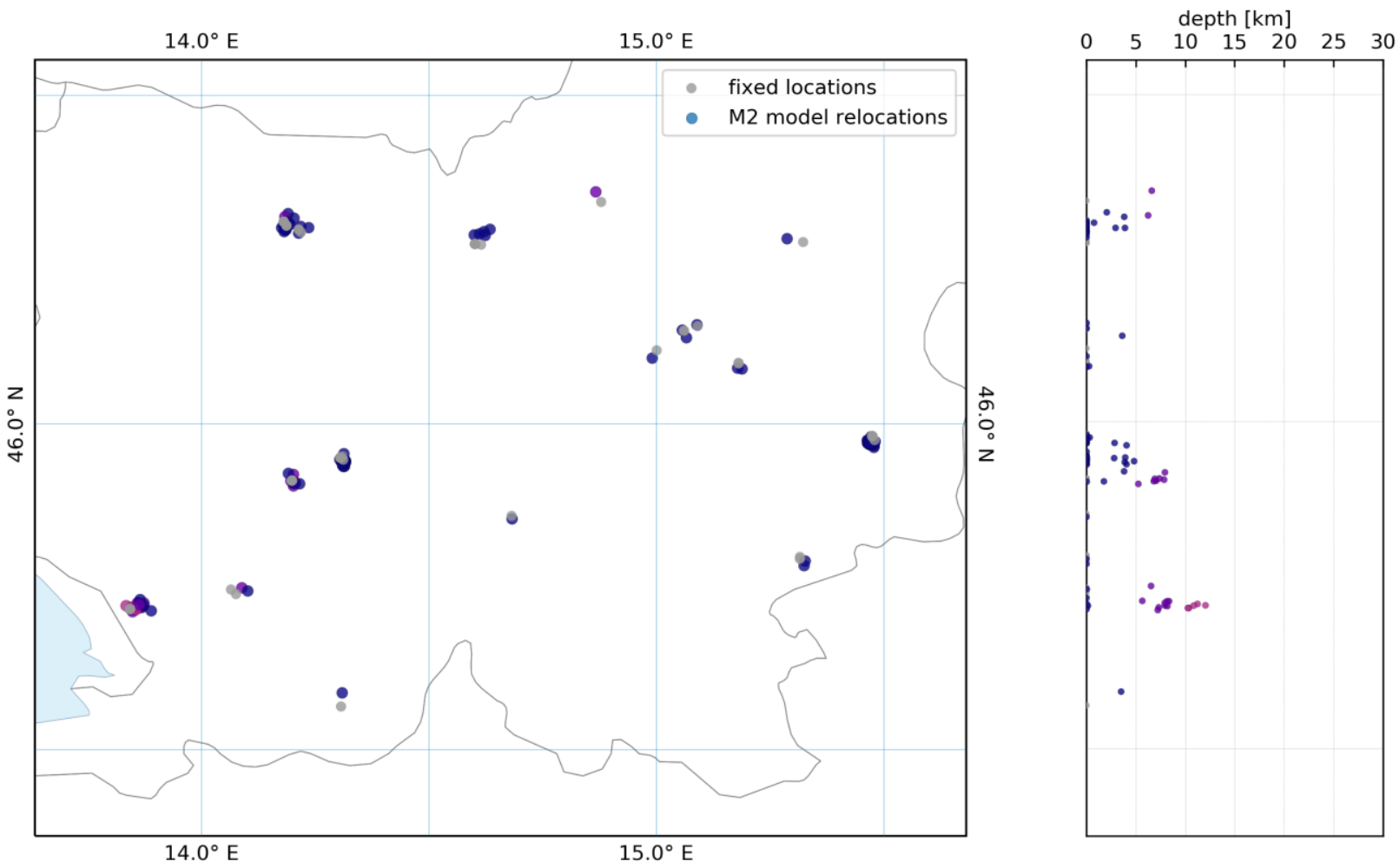

num. of events
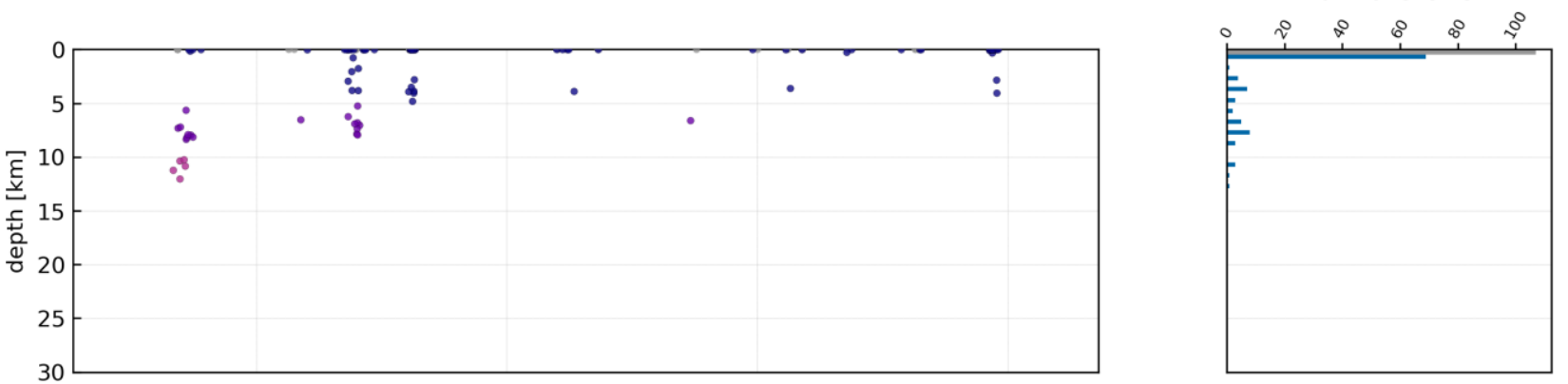

Figure S7: Relocation using the M2 velocity model of 107 blasts with a maximum azimuthal gap of $180^{\circ}$ and at least 8 first $P$ arrival times with uncertainty class of 2 or less. The right and bottom panels show the relocated blasts projected on $\mathrm{N}-\mathrm{S}$ and $\mathrm{W}$ - $\mathrm{E}$ oriented profiles, respectively. The histogram in the lower right corner shows the number of relocated blasts in $1 \mathrm{~km}$ depth bins. 
Table S8: Velocities and statistics obtained for the NW subregion.

\begin{tabular}{|l|l|l|l|l|}
\hline Depth $[\mathrm{km}]$ & Median $[\mathrm{km} / \mathrm{s}]$ & 15 th perc. $[\mathrm{km} / \mathrm{s}]$ & 85 th perc. $[\mathrm{km} / \mathrm{s}]$ & Delta perc. $[\mathrm{km} / \mathrm{s}]$ \\
\hline-4 & 5.74 & 5.37 & 5.81 & 0.44 \\
\hline 0 & 5.75 & 5.37 & 5.81 & 0.44 \\
\hline 2 & 5.75 & 5.56 & 5.83 & 0.27 \\
\hline 4 & 5.75 & 5.72 & 5.92 & 0.2 \\
\hline 6 & 5.82 & 5.74 & 6.03 & 0.29 \\
\hline 8 & 6.04 & 6.01 & 6.09 & 0.08 \\
\hline 10 & 6.08 & 6.04 & 6.13 & 0.09 \\
\hline 12 & 6.13 & 6.09 & 6.15 & 0.06 \\
\hline 14 & 6.13 & 6.1 & 6.16 & 0.06 \\
\hline 16 & 6.15 & 6.12 & 6.19 & 0.07 \\
\hline 18 & 6.21 & 6.15 & 6.27 & 0.12 \\
\hline 20 & 6.22 & 6.17 & 6.32 & 0.15 \\
\hline 23 & 6.36 & 6.19 & 6.51 & 0.32 \\
\hline 26 & 6.45 & 6.38 & 6.75 & 0.37 \\
\hline 30 & 6.72 & 6.45 & 6.92 & 0.47 \\
\hline 34 & 6.91 & 6.54 & 7.02 & 0.48 \\
\hline 38 & 7.18 & 6.57 & 7.58 & 1.01 \\
\hline 42 & 7.8 & 7.2 & 8.05 & 0.85 \\
\hline 46 & 8.05 & 7.36 & 8.22 & 0.86 \\
\hline 50 & 8.1 & 7.5 & 8.24 & 0.74 \\
\hline & & & & \\
\hline
\end{tabular}


Table S9: Velocities and statistics obtained for the SW subregion.

\begin{tabular}{|l|l|l|l|l|}
\hline Depth $[\mathrm{km}]$ & Median $[\mathrm{km} / \mathrm{s}]$ & 15 th perc. $[\mathrm{km} / \mathrm{s}]$ & 85 th perc. $[\mathrm{km} / \mathrm{s}]$ & Delta perc. $[\mathrm{km} / \mathrm{s}]$ \\
\hline-4 & 5.28 & 4.9 & 5.81 & 0.91 \\
\hline 0 & 5.76 & 5.59 & 6 & 0.41 \\
\hline 2 & 5.92 & 5.63 & 6.07 & 0.44 \\
\hline 4 & 5.94 & 5.83 & 6.07 & 0.24 \\
\hline 6 & 5.97 & 5.88 & 6.09 & 0.21 \\
\hline 8 & 6.02 & 5.95 & 6.09 & 0.14 \\
\hline 10 & 6.04 & 5.97 & 6.1 & 0.13 \\
\hline 12 & 6.05 & 5.97 & 6.19 & 0.22 \\
\hline 14 & 6.16 & 6.02 & 6.24 & 0.22 \\
\hline 16 & 6.16 & 6.06 & 6.25 & 0.19 \\
\hline 18 & 6.17 & 6.08 & 6.26 & 0.18 \\
\hline 20 & 6.17 & 6.08 & 6.37 & 0.29 \\
\hline 23 & 6.4 & 6.09 & 6.52 & 0.43 \\
\hline 26 & 6.52 & 6.35 & 6.65 & 0.3 \\
\hline 30 & 6.7 & 6.56 & 6.79 & 0.23 \\
\hline 34 & 6.85 & 6.56 & 6.89 & 0.33 \\
\hline 38 & 7.23 & 6.57 & 7.41 & 0.84 \\
\hline 42 & 7.6 & 7.22 & 7.82 & 0.6 \\
\hline 46 & 7.97 & 7.36 & 8.22 & 0.86 \\
\hline 50 & 8.1 & 7.5 & 8.24 & 0.74 \\
\hline & & & & \\
\hline & & & & \\
\hline
\end{tabular}


40 Table S10: Velocities and statistics obtained for the E subregion.

\begin{tabular}{|c|c|c|c|c|}
\hline Depth [km] & Median $[\mathrm{km} / \mathrm{s}]$ & 15 th perc. $[\mathrm{km} / \mathrm{s}]$ & 85th perc. $[\mathrm{km} / \mathrm{s}]$ & Delta perc. $[\mathrm{km} / \mathrm{s}]$ \\
\hline-4 & 4.89 & 4.59 & 5.19 & 0.6 \\
\hline 0 & 5.46 & 5.43 & 5.54 & 0.11 \\
\hline 2 & 5.46 & 5.44 & 5.55 & 0.11 \\
\hline 4 & 5.63 & 5.53 & 5.75 & 0.22 \\
\hline 6 & 5.7 & 5.62 & 5.86 & 0.24 \\
\hline 8 & 5.93 & 5.9 & 5.95 & 0.05 \\
\hline 10 & 5.95 & 5.91 & 5.99 & 0.08 \\
\hline 12 & 6.03 & 5.99 & 6.05 & 0.06 \\
\hline 14 & 6.04 & 6 & 6.08 & 0.08 \\
\hline 16 & 6.07 & 6.01 & 6.13 & 0.12 \\
\hline 18 & 6.1 & 6.02 & 6.16 & 0.14 \\
\hline 20 & 6.16 & 6.02 & 6.27 & 0.25 \\
\hline 23 & 6.36 & 6.09 & 6.4 & 0.31 \\
\hline 26 & 6.4 & 6.28 & 6.57 & 0.29 \\
\hline 30 & 6.64 & 6.45 & 6.85 & 0.4 \\
\hline 34 & 6.91 & 6.54 & 7.01 & 0.47 \\
\hline 38 & 7.18 & 6.57 & 7.57 & 1 \\
\hline 42 & 7.8 & 7.2 & 8.06 & 0.86 \\
\hline 46 & 8.07 & 7.36 & 8.22 & 0.86 \\
\hline 50 & 8.1 & 7.5 & 8.24 & 0.74 \\
\hline
\end{tabular}

